International Journal of Modern Physics C

Vol. 31, No. 3 (2020) 2050046 (11 pages)

(C) World Scientific Publishing Company

DOI: $10.1142 / \mathrm{S} 0129183120500461$

\title{
Study on turbulence drag reduction of riblet plate in hypersonic turbulent flows
}

\author{
Hao Zhou*, Xinliang Li and Changping $\mathrm{Yu}^{\dagger}$ \\ LHD, Institute of Mechanics \\ Chinese Academy of Sciences \\ Beijing 100190, P. R. China \\ School of Engineering Science \\ University of Chinese Academy of Sciences \\ Beijing 100049, P. R. China \\ *95574805@qq.com \\ †cpyu@imech.ac.cn
}

Received 22 October 2019

Accepted 9 December 2019

Published 30 January 2020

\begin{abstract}
This paper focuses on turbulence drag reduction of riblet plate in hypersonic turbulent flows. We use direct numerical simulation (DNS) and large eddy simulation (LES) to simulate threedimensional spatially-developing boundary layer over the flat plate and riblet plate with a freestream Mach number $M a=6$. The results reveal the influence of different riblet heights $h_{w}$ and riblet distances $\lambda_{w}$ on drag reduction effect. The drag reduction effect increases with the increase of riblet height and the decrease of riblet distance within suitable range of parameter values. Through analysis, it can be seen that the riblet plate affects the turbulent contribution of the skin friction by suppressing or destroying the large-scale vortex structure. Combined with the actual engineering design requirements, we can use the riblet plate with appropriate parameters to achieve the purpose of turbulence control.
\end{abstract}

Keywords: Hypersonic; turbulence control; numerical simulation; riblet plate.

PACS No.: 47.85.Gj.

\section{Introduction}

In the design of the vehicle, the skin friction drag is one of the important sources of total drag. Reducing the skin friction drag can save energy, or allow aircraft to fly longer distances while consuming the same amount of energy. Drag reduction by changing or controlling the state of the fluid (including mechanical states, such as velocity of motion; or thermodynamic states, such as temperature) is called drag reduction control. In practical problems, the boundary layer is generally in a turbulent state, so it is of great significance to study the control of turbulent boundary

†Corresponding author. 
layer drag reduction. In supersonic flow, skin friction drag accounts for about $30 \%$ of the total drag. It is a hot topic of compressible turbulence research to reduce drag and control compressible turbulence. At present, turbulence drag reduction control is divided into active control and passive control according to whether energy is input into the flow field. Active control requires energy input into the flow, such as vibration of the wall surface, adding volume force to the flow field, blowing on the wall surface, or plasma input to the flow field. Passive control does not require the input of energy into the flow field, such as using flexible boundaries, polymers to change fluid behavior, or using riblet plate or vortex-crushing devices. ${ }^{1-7}$ In the flight process of hypersonic aircraft, high heat flow and high friction drag will be generated by wall turbulence, which will seriously affect the flight safety of aircraft. Therefore, it is more urgent to study the control of boundary layer transition and turbulence. The main research content of this paper is to use direct numerical simulation (DNS) and large eddy simulation (LES) to study the hypersonic plate flow with Mach number $M a=6$, study the control mechanism of turbulence reduction and heat transfer reduction through riblet plate, and provide appropriate control parameters to provide support for wind tunnel experiments or actual flight of aircraft. The structure of the paper is as follows: the problem configuration and numerical method are introduced in Sec. 2. The results and discussion are performed in Sec. 3, Finally, the conclusions are given in Sec. 4.

\section{Problem Configuration and Numerical Method}

The compressible Navier-Stokes equations as given as

$$
\begin{gathered}
\frac{\partial \rho}{\partial t}+\frac{\partial \rho u_{j}}{\partial x_{j}}=0 \\
\frac{\partial \rho u_{i}}{\partial t}+\frac{\partial \rho u_{i} u_{j}}{\partial x_{j}}=-\frac{\partial p}{\partial x_{i}}+\frac{\partial \sigma_{i j}}{\partial x_{j}} \\
\frac{\partial E}{\partial t}+\frac{\partial(E+p) u_{j}}{\partial x_{j}}=\frac{\partial u_{i} \sigma_{i j}+q_{j}}{\partial x_{j}}, \\
p=\rho R T .
\end{gathered}
$$

In (1)-(4), $\rho, u_{i}, T, E$ and $R$ denote density, velocity, temperature, total energy, and specific gas constant, respectively. The viscous stress tensor $\sigma_{i j}$ and the heat flux vector $q_{j}$ are given by

$$
\begin{gathered}
\sigma_{i j}=2 \mu(T)\left(S_{i j}-\frac{1}{3} \delta_{i j} S_{k k}\right), \\
q_{j}=\frac{C_{p} \mu(T)}{\operatorname{Pr}} \frac{\partial T}{\partial x_{j}}
\end{gathered}
$$

where $C_{p}, \operatorname{Pr}$ are the specific heat at constant pressure and molecular Prandtl number, $\mu=\frac{1}{\operatorname{Re}} \frac{T^{3 / 2}\left(1+T_{s} / T_{\infty}\right)}{T+T_{s} / T_{\infty}}$ is the molecular viscosity calculated using Sutherland's 
law for given $T_{s}=110.3 \mathrm{~K}, \operatorname{Re}=\rho_{\infty} U_{\infty} L / \mu_{\infty}$ is the Reynolds number, and $S_{i j}=\frac{1}{2} \times$ $\left(\frac{\partial u_{i}}{\partial x_{j}}+\frac{\partial u_{j}}{\partial x_{i}}\right)$ is the strain-rate tensor, where $\infty$ denotes the free-stream quantity.

In this section, we will give a posteriori test with hypersonic spatially developing boundary layer with a free-stream Mach number $M a=6$ and free-stream unit Reynolds number $\mathrm{Re} / \mathrm{mm}=1 \times 10^{4}$, free-stream temperature $T_{\infty}=69 \mathrm{~K}$, and an wall temperature $T_{w}$ of $293.88 \mathrm{~K}$.

In a posteriori test, the governing equations (1)-(4) for DNS and LES of compressible flow are solved by using a high precision nondimensional finite difference solver in cartesian coordinates, which is named Opencfd-SC and developed in our group for the compressible flows. The free-stream velocity, free-stream density, and unit length of mesh are selected as the characteristic velocity, characteristic density and characteristic length. The sixth-order monotonicity-preserving optimized scheme (OMP6) is employed for the discretization of the convective terms, ${ }^{8}$ and the sixth-order central difference scheme for the viscous terms. The discretized equations are temporally integrated using an explicit third-order Runge-Kutta scheme.

The computational domain (see Fig. 1) is bounded by in-flow and out-flow boundaries, a wall boundary, a far-field boundary, and the two boundaries (periodic) in the span-wise direction. The computational domain used in this simulation has a size of $L_{x} \times L_{y} \times L_{z}=1200 \times 32 \times 32(\mathrm{~mm})$ in the stream-wise, wall-normal, and span-wise directions. The surface shape of riblet plate is defined as

$$
y(x, z)=h_{w}(x) \sin \left(2 \pi z / \lambda_{w}\right),
$$

where $h_{w}(x)$ is the riblet height and it is a function of the direction of $x, \lambda_{w}$ is the riblet distance, while $h_{w}(x)=0$ is the flat plate. Sketch of the computational domain

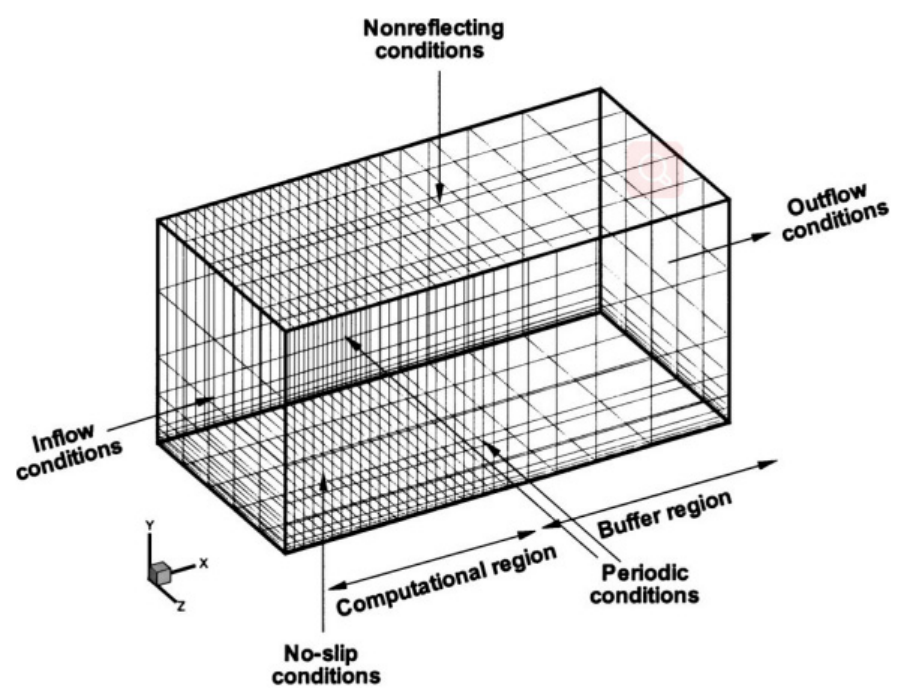

Fig. 1. (Color online) Sketch of the computational domain for the simulation. 

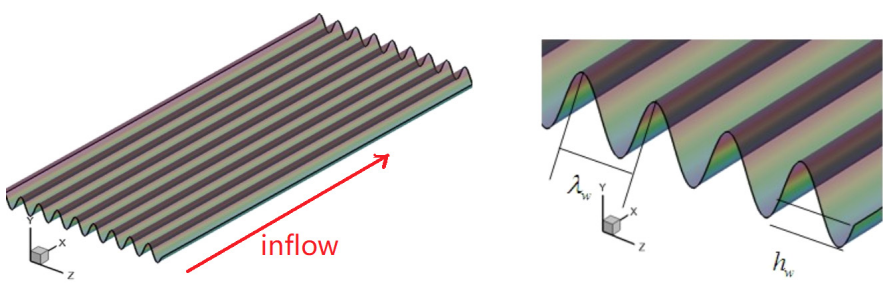

Fig. 2. (Color online) Sketch of the computational domain for riblet plate.

for riblet plate is shown in Fig. 2. As our main concern is the heat/drag reduction in the turbulent region, and in order to save computing resources, we added a region of blowing and suction disturbance $\left(x_{a}<x<x_{b}\right)$ at the leading edge of the plate to ensure the occurrence of transition.

The normal velocity component of the wall surface in the blowing and suction disturbance region $\left(x_{a}<x<x_{b}\right)$ is calculated as

$$
v(x, z, t)=A u_{\infty} f(x) g(z) h(t), \quad x_{a}<x<x_{b},
$$

with $A=0.2$ is the amplitude of the disturbance, $u_{\infty}$ is the free-stream velocity, and

$$
\begin{gathered}
f(x)=4 \sin \theta(1-\cos \theta) / \sqrt{27}, \\
\theta=2 \pi\left(x-x_{a}\right)\left(x_{b}-x_{a}\right), \\
g(z)=\sum_{l=1}^{l_{\max }} Z_{l} \sin \left(2 \pi l\left(z / L_{z}+\phi_{l}\right)\right), \\
\sum_{l=1}^{l_{\max }} Z_{l}=1, \quad Z_{l}=1.25 Z_{l+1}, \\
\left.h(t)=\sum_{m=1}^{m_{\max }} T_{m} \sin \left(\beta t+2 \pi \phi_{m}\right)\right), \\
\sum_{m=1}^{m_{\max }} T_{m}=1, \quad T_{m}=1.25 T_{m+1} .
\end{gathered}
$$

The locations $x_{a}$ and $x_{b}$ indicate the beginning and the end of the blowing and suction zone, respectively, $L_{z}$ is the size of the computational domain in the span-wise direction, $\beta=75000 \mathrm{~Hz}$ is the fundamental frequency of the disturbance, and $\phi_{l}$ and $\phi_{m}$ are random numbers ranging between 0 and 1 . At the same time, for better comparability of simulation results, the upstream of computational domain is flat plate and downstream of computational domain is riblet plate (see Fig. 3).

For the riblet plate, we make orthogonal processing to ensure the correct calculation in the near-wall region. The schematic diagram of the mesh is shown in Fig. 4. 


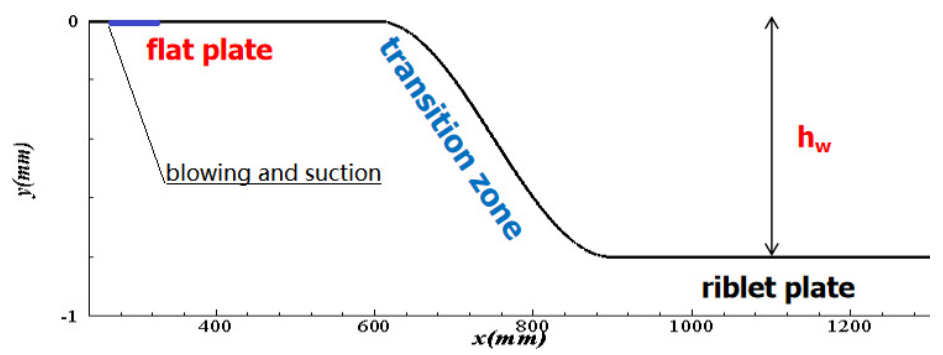

Fig. 3. (Color online) Change of $h_{w}(x)$ in the direction of $x$.
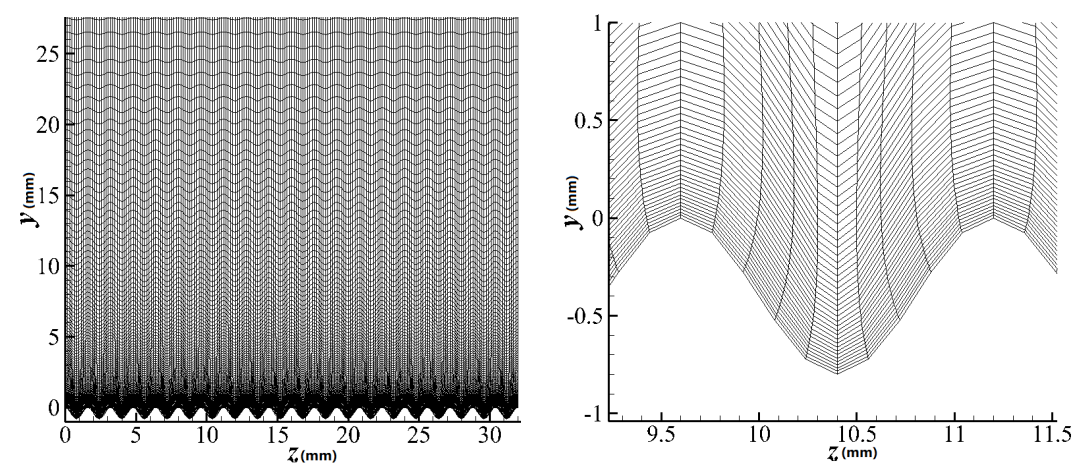

Fig. 4. Locally enlarge the schematic diagram of the grid.

\section{Results and Discussions}

Due to the DNS requires a lot of computational resources, the implicit large eddy simulation (ILES) is adopted to explore the parametric influence in the initial stage of studying the turbulence drag reduction control of the riblet plate.

\subsection{The LES results}

At the initial stage of this research, we used the ILES to study the effects of different parameters $\left(h_{w}\right.$ and $\left.\lambda_{w}\right)$. The total structured nodes number of LES mesh is $N_{x} \times N_{y} \times N_{z}=2210 \times 120 \times 201$, and $\Delta x^{+} \times \Delta y^{+} \times \Delta z^{+}=17.14 \times 0.53 \times 5.69$.

First, we test the effect of riblet height $h_{w}$ on turbulence drag reduction control. The specific method is to fix the riblet distance as $\lambda_{w}=1.6 \mathrm{~mm}$ and obtain the skin friction coefficient $C_{f}$ along the $x$-direction at different riblet heights $h_{w}$ through numerical simulation.

The skin friction coefficient $C_{f}$ is defined as

$$
C_{f}=\mu_{w}\left(\frac{\partial u}{\partial y}\right)_{w} /\left(\frac{1}{2} \rho_{\infty} u_{\infty}^{2}\right)
$$

where $w$ denotes the quantity near the wall region and $\infty$ denotes the free-stream quantity. 


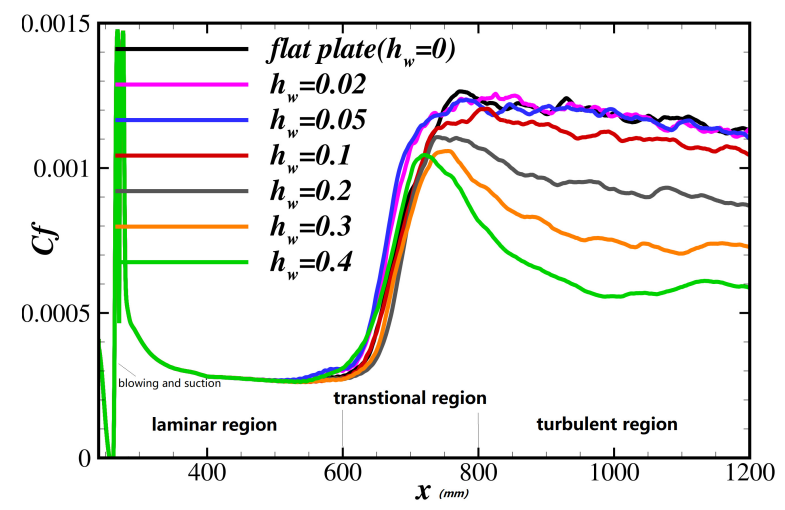

Fig. 5. (Color online) The distribution of skin friction coefficient along the $x$-direction at different heights of riblet plate.

Figure 5 shows the distribution of skin friction coefficient $C_{f}$ along the $x$-direction at different heights $h_{w}$ of riblet plate. It can be seen that the solid black line in Fig. 5 is the global flat plate $\left(h_{w}=0\right)$, after adding blowing and suction disturbance in the region $\left(x_{a}=260<x<280=x_{b}\right)$, the skin-friction of the flat plate (black line) increases quickly when the boundary layer transition begins at approximately $x>600 \mathrm{~mm}$, reaches its maximum at approximately $x>800 \mathrm{~mm}$, and then gradually approaches the steady turbulent value as the boundary layer flow develops into full developed turbulence. That means the flow maintains laminar flow in $0 \leq x \leq 600 \mathrm{~mm}$ region, $600 \leq x \leq 800 \mathrm{~mm}$ is transitional region and $800 \leq$ $x \leq 1200 \mathrm{~mm}$ is the full developed turbulent region. From Fig. 5 , it can be seen that the drag reduction effect is stronger with the increase of height of riblet plate under the fixed distance in turbulent region.

Second, we fixed the height of riblet plate $\left(h_{w}=0.2 \mathrm{~mm}\right)$ and studied the effect of riblet distance $\lambda_{w}$ on turbulent drag reduction. Figure 6 shows the distribution of

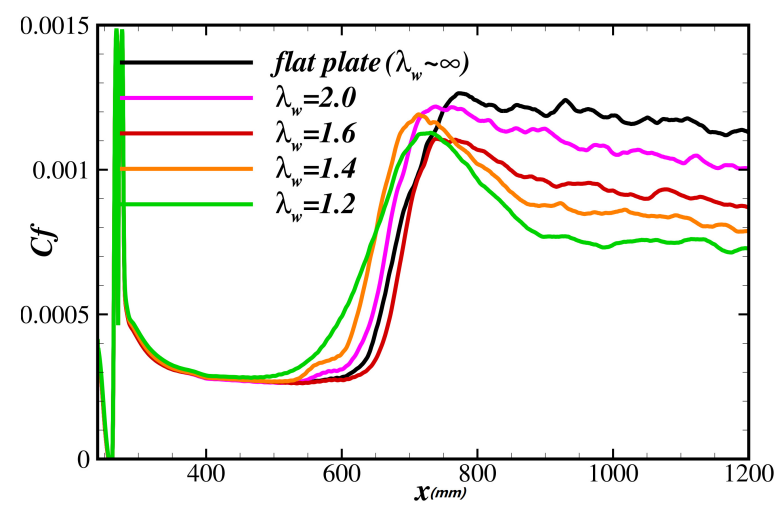

Fig. 6. (Color online) The distribution of skin friction coefficient along the $x$-direction at different distances of riblet plate. 
skin-friction coefficient under different riblet distance $\lambda_{w}$, while riblet distance tends to $\infty$, it can be considered as flat plate. It can been seen from Fig. 6 , at the fixed riblet height, the effect of drag reduction will be enhanced as the riblet distance decreases.

According to Figs. 5 and 6, we have a basic understanding of the turbulent drag reduction mechanism of the riblet plate. With the increase of riblet height and the decrease of riblet distance, the effect of turbulence drag reduction will be enhanced within a certain parameter range. We can choose the right parameters to meet our engineering needs in the design of the aircraft. Since ILES has some computational errors, it can only reveal certain regularity, so, we quantitatively analyze the mechanism of drag reduction by riblet plate through DNS.

\subsection{The DNS results}

Due to the riblet plate is sensitive to the span-wise mesh number, we selected $h_{w}=$ 0.4 and $\lambda_{w}=1.5$ as the working conditions of the riblet plate for DNS, and conducted sufficient encryption on the span-wise direction to ensure the accuracy of calculation. The mesh set for DNS is shown in Table 1.

Figure 7 shows the skin friction coefficient $C_{f}$ along $x$-direction from DNS results of flat plate and riblet plate. It is can be seen that the skin friction of the riblet plate in the turbulent region is less than that of the flat plate, the coefficient is reduced by about $33 \%$.

Table 1. The mesh set for DNS.

\begin{tabular}{lcc}
\hline Mesh & $\Delta x^{+} \times \Delta y^{+} \times \Delta z^{+}$ & $N_{x} \times N_{y} \times N_{z}$ \\
\hline Flat plate & $17.14 \times 0.53 \times 5.69$ & $2210 \times 120 \times 201$ \\
Riblet plate & $8.83 \times 0.53 \times 1.06$ & $3500 \times 120 \times 1051$ \\
\hline
\end{tabular}

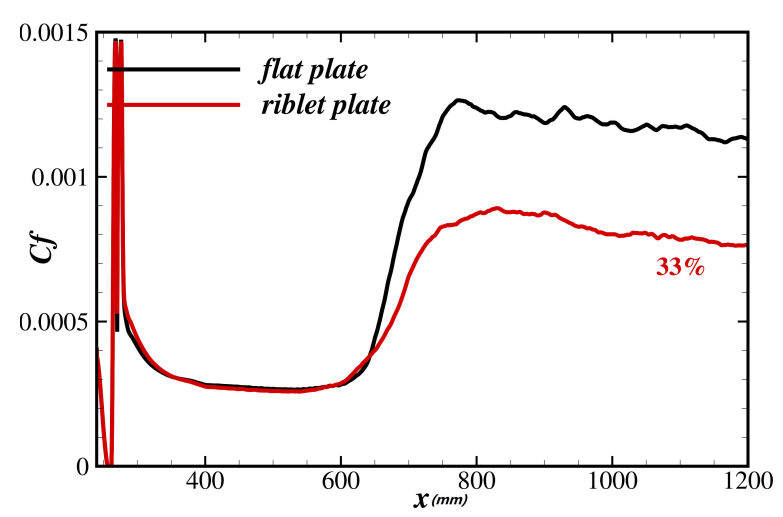

Fig. 7. (Color online) Skin friction coefficient along $x$-direction from DNS results of flat plate and riblet plate. 
The skin heat flux coefficient $S_{t}$ is defined as

$$
S_{t}=k_{w}\left(\frac{\partial T}{\partial y}\right)_{w} / \rho_{\infty} U_{\infty} C_{p}\left(T_{0}-T_{w}\right),
$$

where $w$ denotes the quantity near the wall region and $\infty$ denotes the free-stream quantity, $k$ is the heat transfer coefficient and $T_{0}$ is the free-stream total temperature.

Figure 8 shows the skin heat flux coefficient $S_{t}$ along $x$-direction from DNS results of flat plate and riblet plate. It is can be seen that the skin heat flux of the riblet plate in the turbulent region is less than that of the flat plate, the coefficient is reduced by about $30 \%$.

We display the instantaneous contours of the skin friction coefficient $C_{f}$ of the $x-z$ plane in Fig. 9. It can be seen that there are many large-scale strip structures on the flat plate (Fig. 9(a)). On the contrary, there is no large-scale strip structures on riblet plate (Fig. 9(b)), and the large-scale strip structure is broken by the riblet plate. It can be seen from Fig. $9(\mathrm{~b})$ that the maximum skin friction coefficient $C_{f}$ is

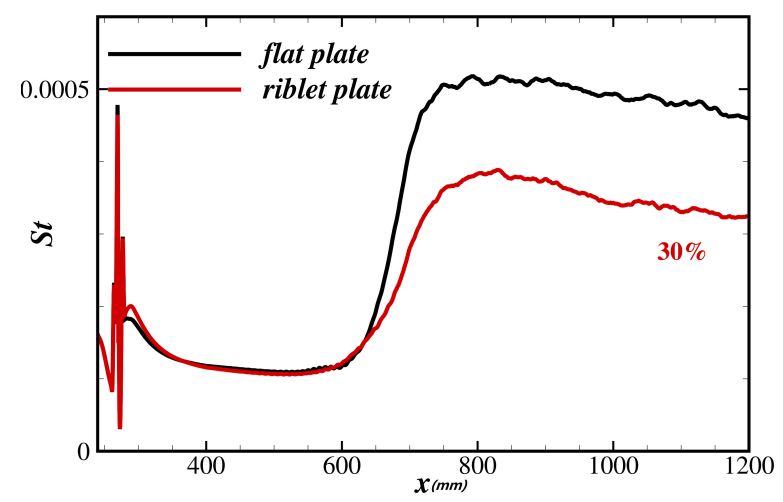

Fig. 8. (Color online) Skin heat flux coefficient along $x$-direction from DNS results of flat plate and riblet plate.

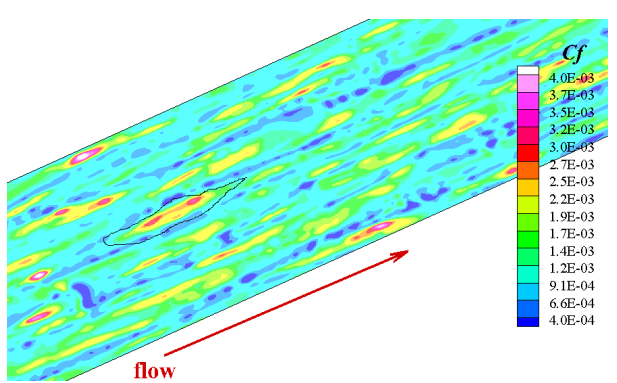

(a)

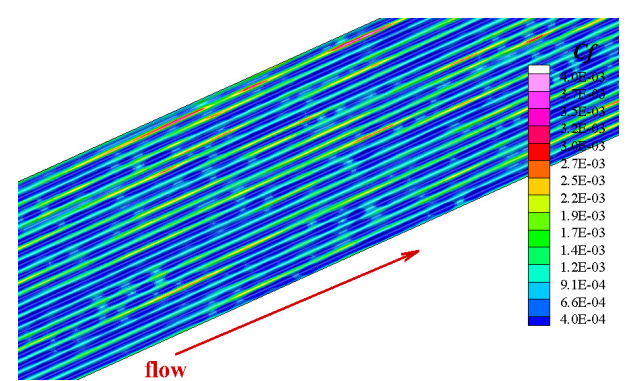

(b)

Fig. 9. (Color online) Instantaneous contours of the skin friction coefficient $C_{f}$ in the $x-z$ plane, (a) flat plate; (b) riblet plate. 


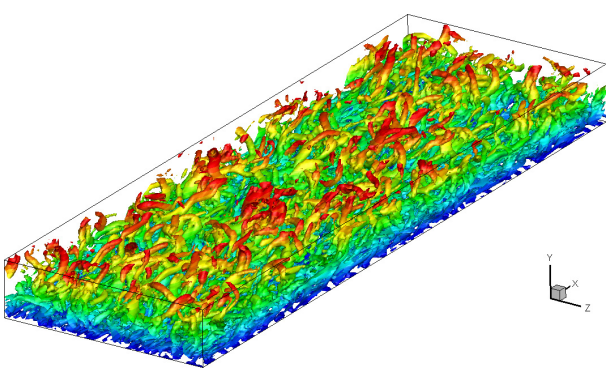

(a)

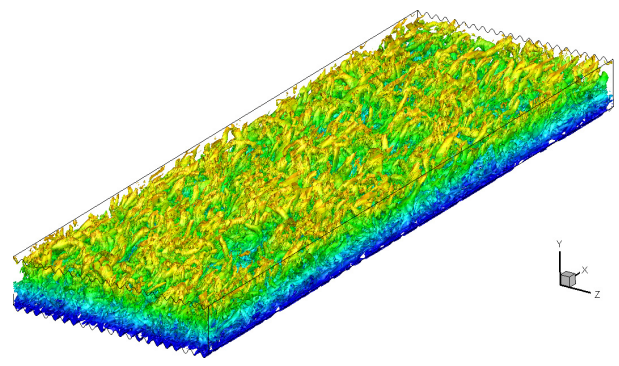

(b)

Fig. 10. (Color online) Isosurface of the $Q$-criterion colored by the stream-wise velocity $u$, (a) flat plate; (b) riblet plate.

distributed at the top of riblet plate, while the minimum skin friction coefficient $C_{f}$ is at the bottom of riblet plate.

Figure 10 shows the coherent vortex structures obtained using the $Q$-criterion for DNS results,${ }^{9}$ which is the isosurface of the discriminant of the velocity gradient tensor. It is a quantity used to identify vortical structures.

$$
\begin{gathered}
Q=-1 / 2\left(S_{i j} S_{i j}-W_{i j} W_{i j}\right) \\
S_{i j}=\frac{1}{2}\left(\frac{\partial u_{i}}{\partial x_{j}}+\frac{\partial u_{j}}{\partial x_{i}}\right), \quad W_{i j}=\frac{1}{2}\left(\frac{\partial u_{i}}{\partial x_{j}}-\frac{\partial u_{j}}{\partial x_{i}}\right) .
\end{gathered}
$$

As can be seen from Fig. 10, there is a big difference between flat plate and riblet plate. There are large-scale coherent vortex structures on flat plate, and on the contrary, there are many broken small-scale vortex structures on riblet plate. This means that the riblet plate has the ability to suppress and destroy large-scale vortex structures, which is also the reason why the riblet plate can reduce turbulence resistance.

Fukagata et al. studied the source of skin friction of channel turbulence in $2002,{ }^{10}$ and proposed the relationship between skin friction coefficient and Reynolds stress and other physical quantities, which was later analyzed by many scholars as Fukagata, Iwamoto and Kasagi (FIK) equation. ${ }^{11}$

$$
\begin{aligned}
C_{f}= & C_{l}+C_{t}+C_{c}+C_{\mathrm{ct}} \\
= & \frac{6}{\operatorname{Re}_{m}}+\frac{6}{\operatorname{Re}_{m}} \int_{0}^{1}(1-y)\left(-\overline{\rho u^{\prime} v^{\prime}}\right) d y \\
& +\frac{6}{\operatorname{Re}_{m}} \int_{0}^{1}(1-y)\left(\mu^{*} \frac{\partial \bar{u}}{\partial y}\right) d y \\
& +\frac{6}{\operatorname{Re}_{m}} \int_{0}^{1}(1-y)\left(\overline{\mu^{\prime}\left(\frac{\partial u^{\prime}}{\partial y}+\frac{\partial v^{\prime}}{\partial x}\right)}\right) d y
\end{aligned}
$$


Table 2. Contribution of skin friction $\left(\times 10^{-3}\right)$.

\begin{tabular}{lccccc}
\hline Case & $C_{f}$ & $C_{t}$ & $C_{c}$ & $C_{\text {ct }}$ & $C_{l}$ \\
\hline M6 flat plate & 1.130 & 0.812 & 0.046 & 0.001 & 0.272 \\
M6 riblet plate & 0.760 & 0.440 & 0.040 & 0.001 & 0.270 \\
\hline
\end{tabular}

where the skin friction $C_{f}$ is divided into four categories: the laminar contribution $C_{l}$, the turbulent contribution (Reynolds stress terms) $C_{t}$, the compressible contribution (compressible terms) $C_{c}$ and the compressible-turbulent interaction term (compressible/turbulent terms) $C_{\text {ct }}$.

It can be seen from Table 2, the turbulent skin friction is the main sources of laminar contribution $C_{l}$ and the turbulent contribution (Reynolds stress terms) $C_{t}$, about contributed about $96 \%$ of the total skin friction, compressible contribution $C_{c}$, compressible and turbulent interaction contribution $C_{\text {ct }}$ contribution to the skin friction can be neglected. Because of the existence of the riblet plate, the turbulence contribution $C_{t}$ which is closely related to the Reynolds stress is affected, thus directly lead to a drop in the turbulent frictional resistance.

\section{Conclusions}

In this paper, we use DNS and LES to research the effects of riblet plate on skin friction in hypersonic plate boundary layer $(\mathrm{Ma}=6)$. The results show that the turbulence drag reduction effect of the riblet plate is stronger with the increase of the riblet height $h_{w}$ and the decrease of the riblet distance $\lambda_{w}$. When the appropriate parameters are selected $\left(h_{w}=0.4 \mathrm{~mm}, \lambda_{w}=1.5 \mathrm{~mm}\right)$, the drag reduction effect can reach $33 \%$ and the heat reduction effect can reach $30 \%$. This provides a reference for the design of aircraft. At the same time, the analysis results show that the existence of riblet plate could destroys the original structure of the near-wall region. In the full developed turbulence, the compressible contribution $C_{C}$ and the compressible and turbulent interaction contribution $C_{\mathrm{ct}}$ are very weak to skin friction, and the turbulent contribution $C_{t}$ is dominant contribution of skin friction. And because the riblet plate weakens the turbulence intensity, the contribution of turbulence $C_{t}$ which is closely related to Reynolds stress to the skin friction decreases.

\section{Acknowledgments}

This work was supported by the National Key Research and Development Program of China (2019YFA0405300 and 2016YFA0401200) and the NSFC Projects (Nos. 91852203, 91441103, 11372330, 11472278), Science Challenge Project (JCKY2016212A501). The authors thank National Supercomputer Center in Tianjin (NSCC-TJ), and National Supercomputer Center in GuangZhou (NSCC GZ) for providing computer time. 


\section{References}

1. H. Choi, P. Moin and J. Kim, J. Fluid Mech. 255, 503 (1993).

2. M. J. Walsh, AIAA J. 21, 485 (1983).

3. X. Li and X. Zhong, J. Spacecr. Rockets 45, 1165 (2008).

4. D. W. Bechert and M. Bartenwerfer, J. Fluid Mech. 206, 105 (1989).

5. M. J. Walsh, Drag Characteristics of V-Groove and Transverse Curvature Riblets (1980).

6. S. S. Saravi and K. Cheng, Eur. Sci. J. 9 (2013).

7. R. Grüneberger and W. Hage, Exp. Fluids 50, 363 (2011).

8. X. Li, Y. Leng and Z. He, Int. J. Numer. Methods Fluids 73, 560 (2013).

9. J. Jeong and F. Hussain, J. Fluid Mech. 285, 69 (1995).

10. K. Fukagata, K. Iwamoto and N. Kasagi, Phys. Fluids 14, L73 (2002).

11. T. Gomez, V. Flutet and P. Sagaut, Phys. Rev. E 79, 035301 (2009). 